\title{
Clinical applications of magnetic resonance imaging in the hand and wrist for soft tissue and ligamentous injuries
}

\author{
Robert B Turner MD FRCSC ${ }^{1}$, Robert S Richards MD FRCSC ${ }^{2}$, James H Roth MD FRCSC FACS ${ }^{3}$ \\ ${ }^{1}$ Department of Surgery, Hand and Upper Limb Centre, St Joseph's Health Centre; ${ }^{2}$ Division of \\ Plastic Surgery, Department of Surgery; ${ }^{3}$ Division of Orthopedic Surgery, Department of Surgery, \\ University of Western Ontario, London, Ontario
}

\begin{abstract}
RB Turner, RS Richards, JH Roth. Clinical applications of magnetic resonance imaging in the hand and wrist for soft tissue and ligamentous injuries. Can J Plast Surg 1998;6(3):141-145.

With advances in magnetic resonance imaging (MRI) technology and observer experience, MRI is an established part of the diagnostic work-up for soft tissue injuries of the hand and wrist. It potentially minimizes the need for invasive procedures such as arthrography or arthroscopy. However, there are limitations of MRI, and many patients who undergo MRI of the wrist still need wrist arthroscopy for both diagnostic and therapeutic purposes. A review of the recent literature of the role of MRI in the assessment of soft tissue injuries of the hand and wrist is presented.
\end{abstract}

Key Words: Ligaments, Magnetic resonance imaging, Wrist

Applications cliniques de l'imagerie par résonance magnétique de la main et du poignet lors d'atteintes des tissus mous et des ligaments

RÉSUMÉ : Grâce aux progrès de la technologie de l'imagerie par résonance magnétique (IRM) et à l'expérience des observateurs, l'IRM fait désormais partie des épreuves diagnostiques utilisées d'emblée lors de blessures des tissus mous à la main et au poignet. Elle offre l'avantage d'un recours moins fréquent aux interventions effractives comme l'arthrographie ou l'arthroscopie. Par contre, l'IRM présente des limites et de nombreux patients qui subissent l'IRM au poignet ont tout de même besoin d'arthroscopie à des fins diagnostiques et thérapeutiques. On présente ici une récente analyse de la littérature sur le rôle de l'IRM dans l'évaluation des blessures des tissus mous de la main et du poignet.

$\mathrm{T}$ he past decade has seen a great leap forward in the technology available for diagnostic imaging of the hand and wrist. The clinician and radiologist are confronted with a variety of imaging techniques. Different algorithms have been published to guide the investigation of wrist disorders. The arrival of wrist arthroscopy and magnetic resonance imaging (MRI) has changed these previous approaches. These investigations offer new information about anatomical abnormalities and may obviate the previous stepladder approach.

Correspondence: Dr Robert S Richards, Hand and Upper Limb Centre, St Joseph's Health Centre, 268 Grosvenor Street, London, Ontario N6A 4L6. Telephone 519-646-6046, fax 519-646-6049, e-mail rrichard@julian.uwo.ca
Arthroscopy remains the gold standard for the diagnosis of intra-articular soft tissue injuries of the wrist. However, as experienced is gained, MRI may become the preferred procedure. It potentially minimizes the need for invasive procedures such as arthrography or arthroscopy.

However, the hand and wrist have complex osseous and soft tissue anatomy. It is hard to determine which studies offer valid clinical information. For example, oblique views of the wrist are part of the standard radiographic examination in many hospitals but rarely add information to well done posteroanterior and lateral films.

Similar difficulties exist with bone scans. Although the sensitivity is excellent, the specificity of the bone scan is poor. Other investigative modalities are essential to interpret the results. 


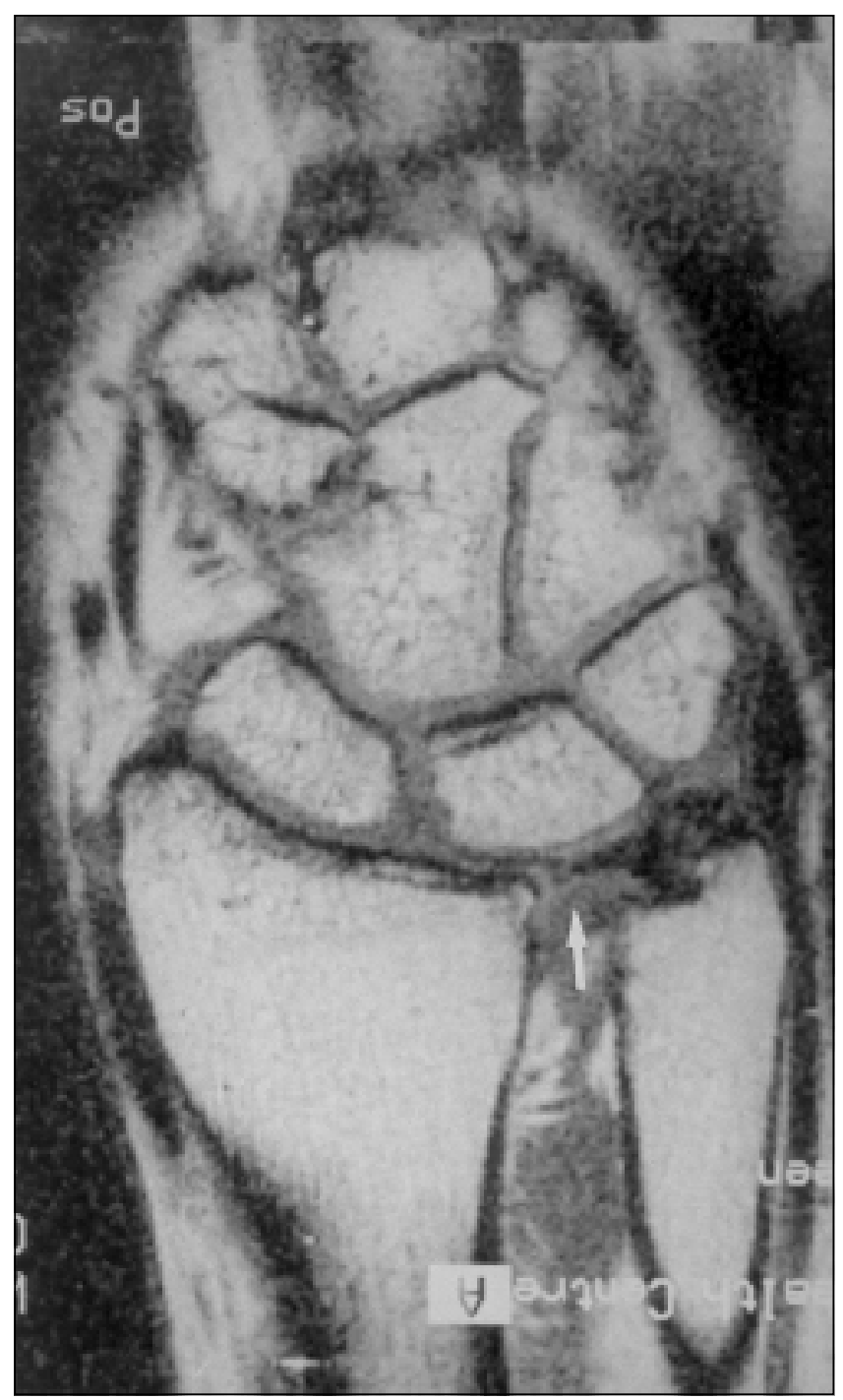

Figure 1) T2 weighted image showing radial triangular fibrocartilage complex tear (arrow)

MRI scans must be interpreted in the context of knowledge gained from open surgery and arthroscopy. Comparison of techniques must be made so that the role of MRI in the investigation of wrist problems can be determined. The role of MRI in the assessment of soft tissue injuries of the hand and wrist is reviewed. The reported accuracy of MRI is compared with current imaging techniques. Treatment of intra-articular ligamentous injuries of the wrist and thumb metacarpal, ulnar collateral ligament (UCL) injuries, chronic wrist pain, carpal tunnel syndrome and infections is reviewed.

\section{INTRA-ARTICULAR WRIST LIGAMENTOUS INJURIES}

\section{Triangular fibrocartilage complex injuries}

The triangular fibrocartilage complex (TFCC) suspends the distal radius and ulnar carpus from the distal ulna. The TFCC functions as the main stabilizer of the distal radioulnar joint and the ulnar carpus (1). A tear or perforation of the TFCC is a common cause of ulnar sided wrist pain.
In the past, investigation of the TFCC was done with arthrographic examination. However, this is invasive and is limited to evaluation of surface defects. Recently, arthroscopy has replaced arthrography, but it is also an invasive procedure. Recent MRI work has shown great promise (2). Correlation of MRI images with histology has shown that MRI can distinguish TFCC degeneration from perforation. Degeneration appears as high signal intensity on $\mathrm{T} 1$ weighted images and as lower signal intensity on T2 images. TFCC perforation demonstrates high signal intensity on T2 images; the difference on T2 images is due to synovial fluid trapped in perforations (Figure 1). The problem with using MRI in the assessment of TFCC tears is the difficulty in detecting associated cartilage erosions. This was confirmed by Cerofolini et al (3) who showed that MRI assessment of TFCC tears failed to detect cartilaginous lesions in all 10 cases studied; the lesions were later seen at arthroscopy.

MRI imaging has had sensitivity and accuracy rates equal to those of conventional radiocarpal arthrography but is less accurate than three compartment arthrography (4). In imaging the TFCC, Cerofolini et al (3) demonstrated an $80 \%$ sensitivity rate with only two false negatives and no false positives in 10 patients. Others have reported sensitivity rates from $72 \%$ to $93 \%(4,5)$. Schweitzer et al (6) attempted to increase the accuracy of MRI by injecting contrast into the radiocarpal joint before imaging. However, there was only a slight increase in the sensitivity, and the conversion of the test into an invasive test has prevented its widespread acceptance. Another disadvantage of MRI arthrography is its inability to perform true motion studies to study marker flow between compartments.

Correlation between the site of the tear on MRI and the surgical site has been excellent (5). Generally, MRI has better anatomical correlation with arthroscopy than with arthrography. The predictive value of MRI in TFCC injuries lies in its high negative predictive value, thus avoiding the need for arthroscopy. Tears or perforations diagnosed on MRI need arthroscopic confirmation and debridement in the symptomatic patient. In the asymptomatic patient MRI findings of a TFCC tear do not warrant arthroscopic investigation.

\section{Scapholunate ligament tears}

Scapholunate ligament tears present in a fashion similar to a scaphoid fracture, with pain, swelling and tenderness over the anatomical snuff box. Initially, plain radiographs may appear normal, and by the time static subluxation is seen, the remaining secondary ligament support has attenuated. At this time the window of opportunity for performing acute ligament repair has been exceeded.

As a result, provocative radiographic testing for scapholunate dissociation has been developed. Tests involve dynamic loading with the fist clenched (7) or distraction in the Johnson test (8). Dynamic loading with the fist clenched forces the capitate into the scapholunate ligament and may cause a dynamic widening in the scapholunate interval. In the Johnson test axial traction applied to the wrist causes a distal migration of the scaphoid with respect to the lunate in the 
presence of a torn scapholunate ligament. As well, arthrography and bone scans have been used to aid in diagnosis. However, none of these tests allows definitive diagnosis. To fill the void, recent attempts have been made in both cadaveric specimens and patients to define the ability of MRI to visualize these ligaments. However, results have been mixed; Schweitzer et al (6) reported a sensitivity of only $25 \%$ and an accuracy of $64 \%$ in assessing the scapholunate ligament. Others have reported up to $90 \%$ accuracy in scapholunate ligament tears (9). Assessment of partial tears was poor, with a sensitivity of only $18 \%$. MRI assessment of interosseous ligament injuries remains observer dependent and varies from institution to institution (Figure 2). In view of these findings, arthroscopy remains the gold standard for the diagnosis of scapholunate ligament tears.

\section{Lunotriquetral ligament tears}

Lunotriquetral tears with an associated volar intercalary segment instability (VISI) pattern have been recognized over the past two decades (10). In the VISI deformity the lunate appears palmar flexed rather than collinear with the radius. A normal radiolunate angle is less than $15^{\circ}$. The static VISI deformity seen on lateral radiographs results from palmar flexion of the lunate as a result of the intact scapholunate ligament and loss of the dorsiflexion force of the triquetrum. Because of the multiple diagnostic possibilities, diagnosis has been difficult (7).

Assessment of lunotriquetral ligament tears with MRI is more difficult than that of scapholunate ligament injuries. This is due to the size and oblique orientation of the ligament to the plane of the coronal images. Still, reported accuracy ranges from $60 \%$ to $80 \%(4,6)$. The quality of MRI of the wrist improves each year with advances in receiver coil technology and imaging sequences. Recently, the variability in morphological and magnetic resonance signal intensity characteristics of the lunotriquetral ligament was defined (11). Familiarity with the normal anatomical variants makes it easier to make an accurate pathological diagnosis. As well, MRI arthrography has been attempted in order to increase the sensitivity of the MRI, but this has resulted in only a minimal increase in accuracy (6) and does not appear to be justified. Use of either MRI arthrography or digital subtraction arthrography to increase accuracy in diagnosing ligament tears is hampered by the procedure's inability to stress the wrist (12). Wrist motion is necessary to show some tears. MRI and arthrography still need arthroscopic confirmation of the ligament disruption and assessment of the cartilage surfaces of the carpal bones.

\section{Gamekeeper's thumb}

Injury to the UCL of the thumb metacarpal joint is common among young athletes. Stener (13) described a lesion in which the adductor aponeurosis interposes between the ruptured ends of the UCL. Although uncommon, it has been felt to be pathognomonic of a complete rupture. The presence of a Stener lesion has been used as an indication for surgery, but the problem has been delineating the extent of injury.

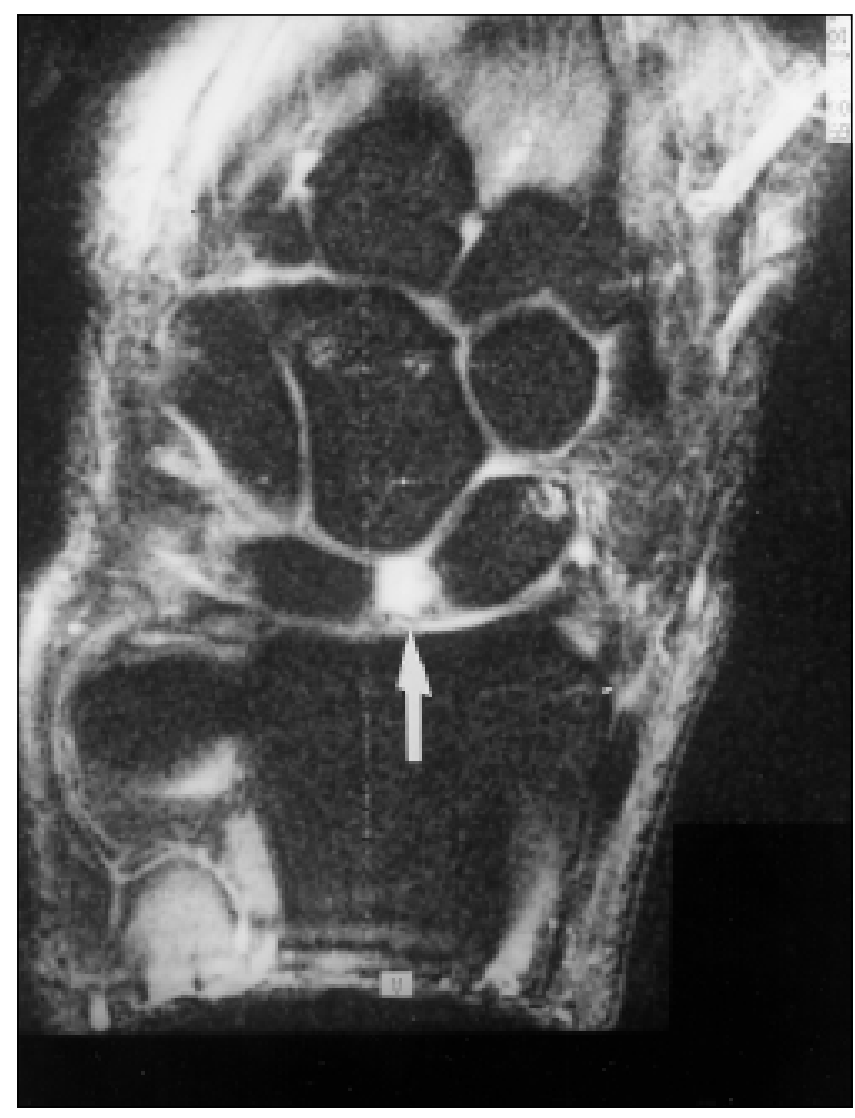

Figure 2) Magnetic resonance image of a scapholunate tear. Note the wide scapholunate gap and the presence of fluid in the scapholunate region (arrow)

Various modalities have been used to diagnose the extent of ligamentous injury. These have included physical examination, arthrography, stress radiography and ultrasound. However, none of these modalities has been able to predict outcome (14). One problem in assessing treatment has been the difficulty in comparing the anatomy between groups treated operatively and nonoperatively. The use of MRI to delineate ligamentous disruption may allow correlation of anatomy with outcome in patients treated nonoperatively. Early attempts have been encouraging. Hergan et al (15), in a study of 17 patients with surgically confirmed ULC tears, were able to depict all UCL tears correctly with $100 \%$ sensitivity and specificity preoperatively. It was also possible to determine the width of the gap between the torn ends of the UCL when it was in an undisplaced position beneath the adductor aponeurosis (15). Figure 3 shows the presence not only of the collateral tear but also of a Stener lesion. The presence of the Stener lesion was later confirmed at surgery and the intraoperative pictures are shown in Figure 4.

\section{Chronic wrist pain}

MRI findings in patients with chronic pain and negative investigations from other modalities are often noncontributory (16). MRI provided added information in only $29 \%$ of patients studied. That study comprised patients with ganglia and carpal tunnel syndrome. A ligamentous or TFCC tear 


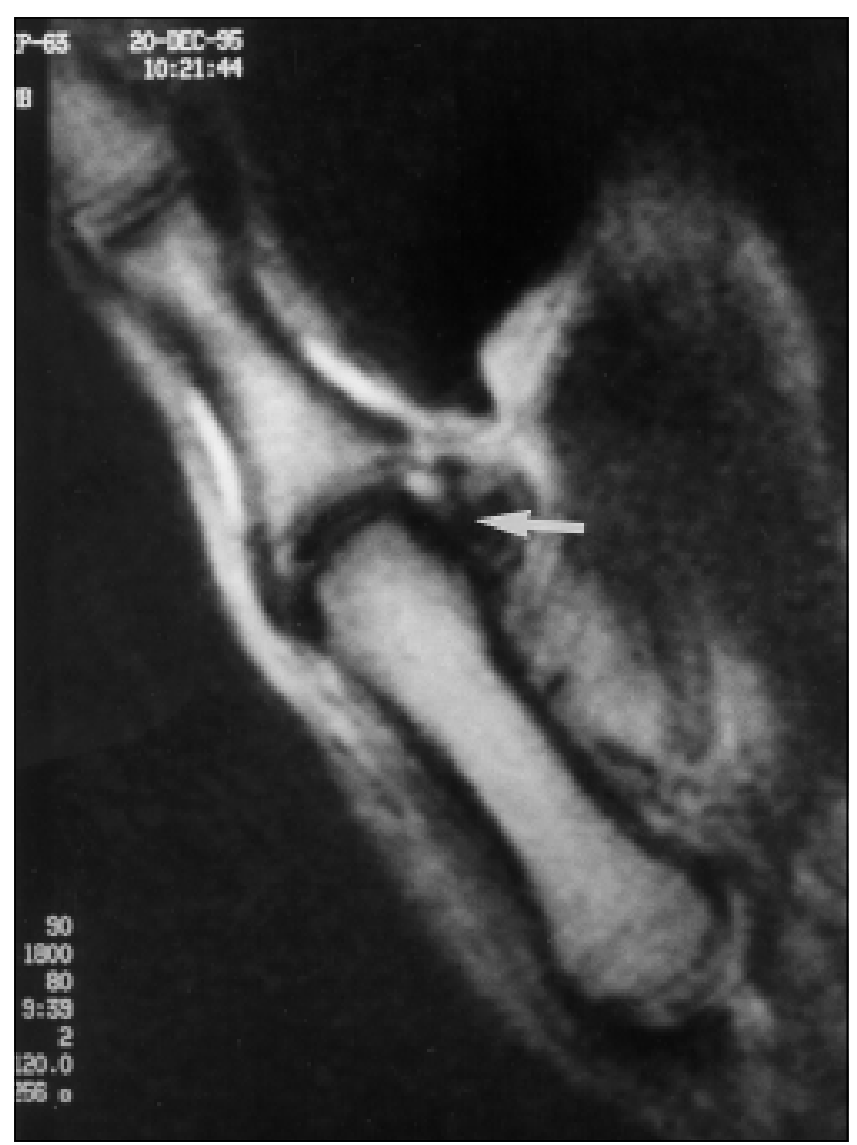

Figure 3) Stener lesion. Arrow indicates the ulnar collateral ligament

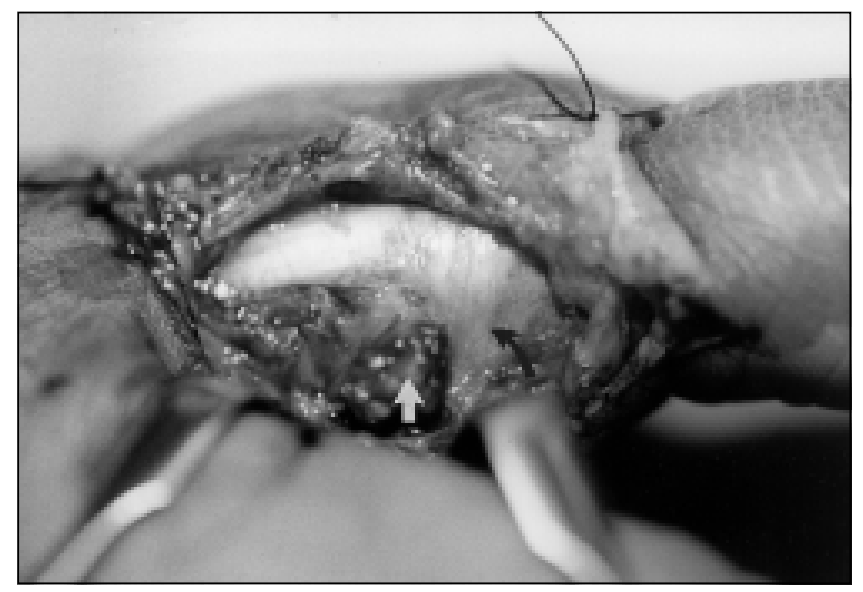

Figure 4) Ulnar collateral ligament (arrow) superficial to the adductor aponeurosis (curved arrow)

was isolated in only one of 41 patients. Thus, the actual value of MRI in chronic pain is low. MRI results in patients with a diagnosis of repetitive forearm strain are also of little value. Little in the way of edema or synovitis can be found in these patients. In contrast, arthroscopy diagnosed pathological lesions in $63 \%$ of chronic wrist pain patients with negative preoperative studies. Many of these were cartilaginous lesions, which MRI defines poorly.
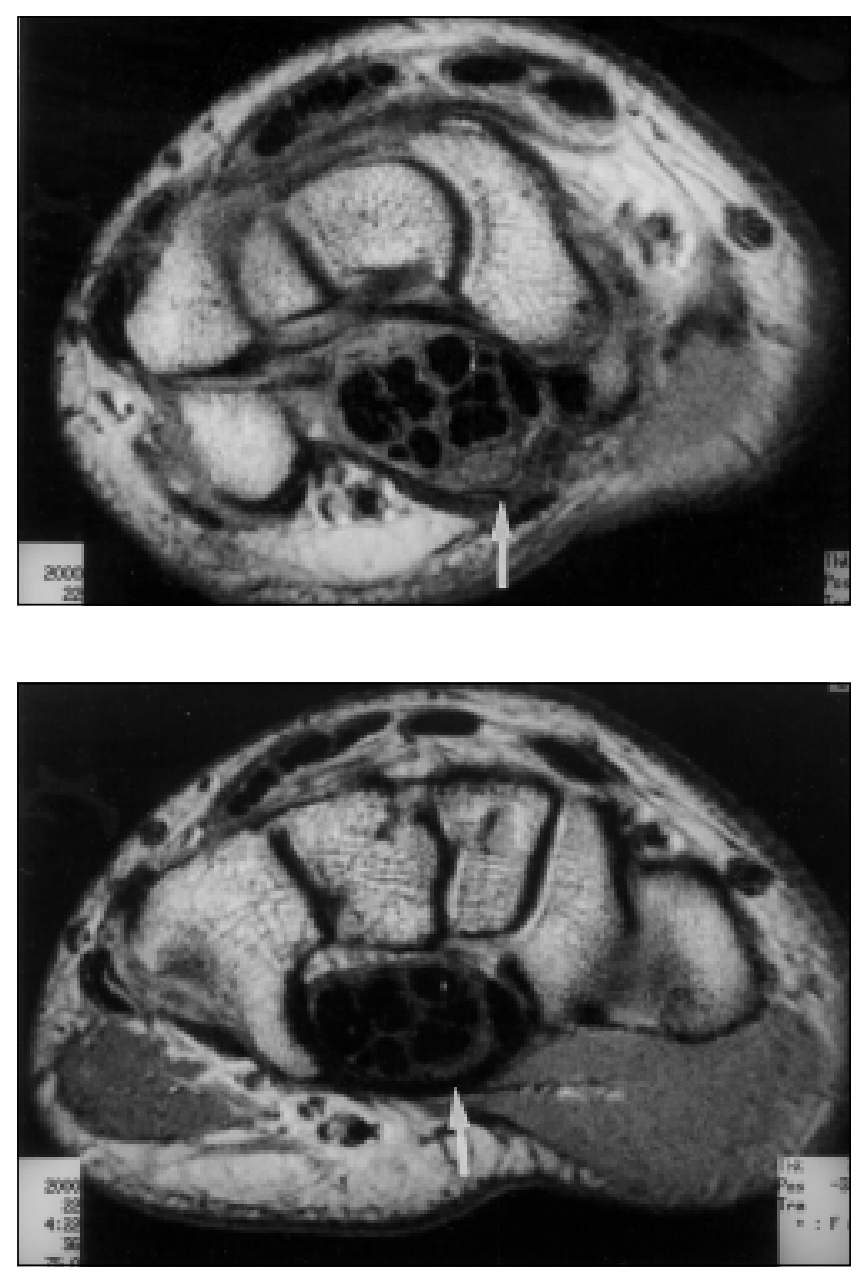

Figure 5) Top Axial image of the carpal tunnel showing the area of fibrosis (arrow) where retinaculum was previously divided. Bottom Axial image just distal to the top image showing an intact carpal ligament

\section{Carpal tunnel syndrome}

The use of MRI to assess the median nerve in the carpal canal has been well described $(17,18)$. MRI has demonstrated both primary compression and inadequate decompression with residual bands of flexor retinaculum (Figure 5). Nerve diameter visualization and assessment of pseudoneuroma formation is not yet possible. As well, attempts have been made to study carpal tunnel volume with changes in wrist position. However, carpal tunnel volume calculations have not been correlated between symptomatic and asymptomatic patients (19).

One major disadvantage is that MRI is not able to distinguish carpal tunnel compression from generalized neural disease or proximal compression. Studies of MRI in carpal tunnel syndrome have not yet established a false negative rate. Thus, a normal MRI would not rule out carpal tunnel syndrome.

\section{Infections}

With respect to acute soft tissue infections of the hand, MRI has been used successfully; however clinically and practically MRI offers little advantage over clinical assessment for 
establishing the necessity of surgical intervention. On the other hand, MRI has been demonstrated to be of value in osteomyelitis. It is extremely sensitive and has been demonstrated to be more effective than ${ }^{111}$ In white blood cell scans, ${ }^{67} \mathrm{Ga}$ scans or ${ }^{99} \mathrm{Tc}$ scans $(20,21)$. Osteomyelitis in hand trauma is uncommon but important to diagnose. MRI imaging may offer an improvement over other techniques.

\section{REFERENCES}

1. Palmer AK. The distal radioulnar joint: anatomy, biomechanics, and triangular fibrocartilage complex abnormalities. Hand Clin 1987;3:31-40.

2. Kang HS, Kindynis $\mathrm{P}$, Brahme SK, et al. Triangular fibrocartilage and intercarpal ligaments of the wrist: MRI imaging. Cadaveric study with gross pathologic and histologic correlation. Radiology 1991;181:401-4.

3. Cerofolini E, Luchetti R, Pederzini L, et al. MRI evaluation of triangular fibrocartilage complex tears in the wrist: comparison with arthrography and arthroscopy. J Comput Assist Tomogr 1990;14:963-7.

4. Gundry CR, Kursunoglu-Brahme S, Schwaighofer B, Kang HS, Sartoris DJ, Resnick D. Is MRI better than arthrography for evaluating the ligaments of the wrist? In vitro study. AJR Am J Roentgenol 1990;154:337-41.

5. Golimbu CN, Firooznia H, Melone CP Jr, Rafii M, Weinreb J, Leber C. Tears of the triangular fibrocartilage of the wrist: MRI imaging. Radiology 1989;173:731-3.

6. Schweitzer ME, Brahme SK, Hodler J, et al. Chronic wrist pain: spin-echo and short tau inversion recovery MR imaging and conventional and MR arthrography. Radiology 1992;182:205-11.

7. Gilula LA, Weeks PM. Post-traumatic ligamentous instability of the wrist. Radiology 1978;129:641-51.

8. Johnson G. Diagnosis of scapholunate dissociation with the traction radiography. Presented at the Manus Canada Annual Meeting, Calgary, June 1 to $2,1991$.

9. Zlatkin MB, Chao PC, Osterman AL, et al. Chronic wrist pain: evaluation with high resolution MRI imaging. Radiology 1989;173:723-9.

10. Taleisnik J. Post traumatic carpal instability. Clin Orthop 1980;149:73-82.

\section{CONCLUSIONS}

MRI is established as a part of the diagnostic work-up for soft tissue injuries of the wrist. Limitations exist, and MRI alone is not sufficient for many traumatic and degenerative lesions. Many patients who undergo MRI of the wrist still need arthroscopy for both diagnostic confirmation and therapeutic purposes.

11. Smith M, Snearly WN. Lunotriquetral interosseous ligament of the wrist: MR appearances in asymptomatic volunteers and arthrographically normal wrists. Radiology 1994;191:199-202.

12. Levinsohn EM. Imaging of the wrist. Radiol Clin North Am 1990;28:905-21.

13. Stener B. Hyperextension injuries to the metacarpophalangeal joint of the thumb. Rupture of ligaments, fracture of sesamoid bones, rupture of flexor pollicis brevis. An anatomical and clinical study. Acta Chir Scand 1963;125:275-93.

14. Pichora DR, McMurtry RY, Bell MJ. Gamekeeper's thumb: a prospective study of functional bracing. J Hand Surg 1989;14:567-73.

15. Hergan K, Mittler C, Oser W. Ulnar collateral ligament: Differentiation of displaced and nondisplaced tears with US and MR imaging. Radiology 1995;194:65-71.

16. Sullivan PP, Berquist TH. Magnetic resonance imaging of the hand, wrist, and forearm: utility in patients with pain and dysfunction as a result of trauma. Mayo Clin Proc 1991;66:1217-21.

17. Mesgarzadeh M, Schneck CD, Bonakdarpour A. Carpal tunnel: MR imaging. Part I. Normal anatomy. Radiology 1989;171:743-8.

18. Mesgarzadeh M, Schneck CD, Bonakdarpour A, Mitra A, Conaway D. Carpal tunnel: MR imaging. Part II. Carpal tunnel syndrome. Radiology 1989;171:749-54.

19. Healy C, Watson JD, Longstaff A, Campbell MJ. Magnetic resonance imaging of the carpal tunnel. J Hand Surg 1990;15:243-8.

20. Beltran J, Noto AM, McGhee RB, Freedy RM, McCalla MS. Infections of the musculoskeletal system: high-field-strength MRI imaging. Radiology 1987;164:449-54.

21. Bassett LW, Gold RH. Magnetic resonance imaging of the muskuloskeletal system: an overview. Clin Orthop 1989;244:17-28. 